\title{
Communication éducative médiatisée : les conditions de la recherche
}

Educative communication through the media : the conditions of research.

\section{Élisabeth Fichez-Vallez}

\section{CpenEdition}

\section{Journals}

Édition électronique

URL : http://journals.openedition.org/edc/2791

DOI : 10.4000/edc. 2791

ISSN : 2101-0366

Éditeur

Université Lille-3

Édition imprimée

Date de publication : 1 mai 1991

Pagination : 53-68

ISSN : $1270-6841$

Référence électronique

Élisabeth Fichez-Vallez, "Communication éducative médiatisée : les conditions de la recherche », Études de communication [En ligne], 12 | 1991, mis en ligne le 15 janvier 2012, consulté le 20 avril 2019. URL : http://journals.openedition.org/edc/2791 ; DOI : 10.4000/edc.2791

Ce document a été généré automatiquement le 20 avril 2019

(c) Tous droits réservés 


\title{
Communication éducative médiatisée : les conditions de la recherche
}

Educative communication through the media: the conditions of research.

\author{
Élisabeth Fichez-Vallez
}

1 L'invention et la mise progressive sur le marché de moyens technologiques radicalement nouveaux affecte depuis quelques années un secteur éducatif confronté à la croissance de plus en plus manifeste des besoins de formation, au niveau des enseignements tant initiaux que continus.

2 Cette situation provoque dans le même temps la multiplication de programmes de recherche-développement initiés à divers échelons (régional, national, européen) par des décideurs auxquels n'échappent pas les enjeux socio-économiques et politiques de la période présente. C'est ainsi que L. Jospin, faisant allusion aux objectifs fixés par la loi d'orientation sur l'éducation de juillet 1989, déclarait un mois plus tard à l'Université d'été de Carcans-Maubuisson, que, pour les atteindre, "il est impératif de tirer parti des technologies modernes d'information et de communication grâce à leur usage pertinent au service de l'éducation ». Dans un autre cadre, le Secrétaire d'Etat à la formation professionnelle, A. Laignel, précisant de son côté les besoins massifs de ce secteur face aux échéances européennes, insistait sur " la nécessaire mise au point d'outils de formation multimédias qui permettent de former plus vite et plus économiquement $»^{1}$.

3 Les appels d'offre lancés ont pour caractéristique générale d'associer formation, démonstration et innovation, à une recherche finalisée, centrée sur une logique d'outils, cette notion devant être prise au sens large, depuis les outils conceptuels et d'analyse jusqu'aux systèmes techniques et aux matériels. Une telle logique induit la problématique « des Nouvelles Technologies Éducatives » (N.T.E.), qui se fonde en gros sur l'idée suivante : une inéluctable mise en adéquation du système de formation doit s'opérer aujourd'hui avec les nouvelles technologies de traitement de l'information et de la communication, 
lesquelles, après avoir déjà eu un impact très important sur les activités économiques et sur les loisirs, vont transformer les différents modes d'apprentissage.

4 Nous avons volontairement eu recours, pour le titre de cet article, à la formule « communication éducative médiatisée ", pour marquer notre distance avec la problématique des N.T.E. Celle-ci nous paraît en effet trop peu s'intéresser, dans les transformations en cours, au développement concomitant de méthodes de gestion empruntées aux modes de fonctionnement industriels, dans des institutions qui, jusque là, ( en particulier dans l'enseignement initial), privilégiaient d'autres modes, liés à un fonctionnement largement plus artisanal, sous la dépendance de financements majoritairement publics. Le processus de technologisation tend notamment à entraîner avec lui la pénétration d'une idéologie managériale dans l'appareil éducatif, le souci -

d'une rentabilisation et d'une optimisation des ressources, la mise en oeuvre d'une rationalisation plus poussée des moyens en vue des objectifs à atteindre. Tout un champ professionnel et social se trouve donc engagé, du fait de la médiatisation, dans une mutation en profondeur (bien qu'encore peu apparente) des pratiques de gestion et de communication qui caractérisaient sa culture et ses institutions, et les rapports de ces dernières avec les autres structures sociales ${ }^{2}$.

5 La recherche dans le secteur éducatif a à prendre en compte une telle mutation et à affirmer ainsi sa dimension critique. Cela ne signifie pas rester à l'écart de tout programme à visée opérationnelle - nous serons au contraire amenée par la suite à montrer que ceux-ci constituent un support obligé. Il s'agit plutôt de mettre en question le présupposé réducteur qui, trop souvent, les inspire, à savoir la simple mise en adéquation du secteur éducatif avec la modernité.

6 Nous voudrions montrer dans cet article que l'orientation de recherche que nous privilégions n'est pas facile à mettre en œuvre dans la situation actuelle ${ }^{3}$. Elle se heurte en effet à au moins deux types de difficultés : d'une part l'exigence de performativité requise par les acteurs institutionnels en position de financer les recherches, d'autre part les contraintes résultant de l'instabilité des projets d'expérimentation, qui constituent les supports empiriques nécessaires à l'avancée de la réflexion théorique.

\section{L'exigence de performativité de la recherche.}

7 Cette expression est empruntée à $\mathrm{F}$. Lyotard qui, dans le cadre d'une réflexion sur la pragmatique du savoir scientifique, montre comment le critère d'efficience des recherches s'est progressivement affirmé depuis la fin du XVIII ${ }^{e}$ siècle, et sert aujourd'hui à justifier la répartition des crédits entre les laboratoires :

La ventilation des fonds de recherche par les Etats, les entreprises et les sociétés mixtes obéit à une logique de l'accroissement de puissance. Les secteurs de la recherche qui ne peuvent pas plaider leur contribution, serait-elle indirecte, à l'optimisation des performances $d u$ système, sont abandonnées par les flux de crédits et voués à la sénescence (1979, p.78).

\section{Le « marché des subventions-recherche ».}

8 La formation médiatisée semble bien être actuellement un secteur appelé à contribuer à une telle optimisation, et, à ce titre, elle bénéficie de crédits incitateurs. Trois niveaux d'intervention, donnant lieu souvent à des participations financières croisées, sont repérables dans ce « marché de subventions-recherche ». 
9 - Un niveau d'intervention à l'échelle européenne : il s'agit d'un ensemble de programmes (DELTA, RACE, COMETT) lancés par la Commission des Communautés depuis quelques années, et donnant lieu régulièrement à des appels d'offre, au fur et à mesure de leur avancement. Chacun d'eux est axé sur une finalité propre, mais ils sont complémentaires (entre eux et avec des programmes nationaux), et visent à développer la conception et la production de systèmes et d'équipements d'apprentissage en dépassant le cadre étroit des marchés nationaux ${ }^{4}$.

10 - Un niveau d'intervention national : l'État joue un rôle important, à la fois d'incitateur et de régulateur, à travers des appels d'offre qui cherchent à cristalliser les énergies autour d'orientations fédératrices. Ceux-ci concernent tout autant le secteur de la formation initiale (F.I.) que celui de la formation continue (F.C.). C'est ainsi qu'en 1987 a été lancé, à l'initiative de la Délégation à la Formation Professionnelle, un important programme pour la conception d'outils de formation multimédias. L'objectif est :

d'impulser un marché de formations multimédias individualisées, qui se rentabilisera à terme par les règles du marché, mais qui a besoin au départ d'une incitation des pouvoirs publics (A. Ramoff, Délégué à la Formation Professionnelle, 1990, p. 9).

11 A ce même échelon, on soulignera le rôle actif de la DATAR qui suscite et finance des projets innovants, destinés à favoriser le développement et l'usage des réseaux et services issus des Nouvelles Technologies de Communication (N.T.C.) dans le cadre de l'aménagement du territoire ${ }^{5}$.

12 - Un niveau d'intervention régional qui se concrétise essentiellement à travers les politiques définies dans le cadre des contrats de plan/recherche État-Région. Certaines régions ont choisi de retenir la formation médiatisée par les N.T.C. dans leurs priorités : $c^{\prime}$ est le cas par exemple du Conseil Régional du Languedoc-Roussillon ${ }^{6}$; c'est également le cas du Conseil Régional du Nord - Pas-de-Calais qui a retenu le thème " Outils et méthodes pour la communication avancée ».

13 Tous ces programmes, qui mettent en jeu des investissements importants ${ }^{7}$, et qui ont vocation à créer entre eux une certaine synergie, sont liés à des enjeux socioéconomiques clairement affichés : relever le défi de la concurrence internationale avec l'Amérique du Nord et le Japon en créant un marché à l'échelle européenne ; viser le marché européen avec les formations multimédias en en faisant un bien exportable ${ }^{8}$; conquérir une image de modernité et d'excellence dans le domaine des technologies éducatives de pointe pour les Régions; enfin, de façon générale, faire face à la nécessaire élévation du niveau des qualifications requise par l'évolution du marché de l'emploi.

Cette offre suscite en retour l'intérêt de nombreux opérateurs : équipes universitaires ou inter-universitaires, au sein desquelles se modifient sensiblement les modes d'organisation traditionnels de la recherche. Un travail de collaboration s'instaure en effet entre chercheurs, formateurs et concepteurs de systèmes et de matériels, ce qui implique un partenariat avec des entreprises du secteur productif. Elle concerne également de grands opérateurs publics capables de mobiliser des moyens importants, tels que l' A.F.P.A., le C.N.E.D., le C.N.D.P. ou le C.N.A.M., ou encore des instituts spécialisés tels que l'IDATE à Montpellier.

14 Cet état des lieux inciterait à penser que la demande sociale de recherche dans le secteur de la formation médiatisée est largement satisfaite par cette offre. Pourtant, notre propre expérience nous amène à dire que certains sous-secteurs sont assez peu ou mal investis ; de même nous constatons que la recherche à visée critique a des difficultés à affirmer sa 
légitimité sociale à cause de l'exigence de performativité qui est requise des programmes de recherche-développement.

\section{Inégalité de traitement de la demande sociale de recherche.}

Le sous-secteur qui nous parait le plus révélateur de cette inégalité de traitement est l'enseignement initial. Non pas qu'il soit laissé à l'écart de tout programme : les bulletins de l'IDATE (cf. en particulier ceux de 1986 et 1989) témoignent des études menées par les chercheurs de cet institut à propos des établissements câblés dans diverses régions de France, sur commande de la DATAR (c'est le cas pour le suivi de l'expérimentation « Ulysse » à Lézignan, promue par celle-ci), ou sur commande de collectivités territoriales. Mais d'autres opérations, ne bénéficiant pas de l'appui financier de tels commanditaires, posent problème pour leur suivi. C'est notamment le cas de la chaîne autoprogrammable "Educâble », expérimentée à Montpellier avec le concours de la ville et du C.R.D.P. local qui a été chargé du développement du produit. Avant que cette télévidéothèque ne soit mise en service dans d'autres sites ${ }^{9}$, une première étude d'évaluation, commandée à l'IDATE et financée par l'Agence du Câble, a été réalisée à la fin de la première année de fonctionnement à Montpellier. Il est fait allusion dans cette étude à la réponse qu'Educâble semble pouvoir apporter à la sous-utilisation pédagogique du matériau audiovisuel en formation initiale (cf. Rabaté, 1988, p. 60). Une telle hypothèse, fondée sur l'accessibilité du document audiovisuel grâce au câble, méritait d'être vérifiée : est-ce aujourd'hui cette disponibilité qui permettra à l'audiovisuel de se développer dans les pratiques pédagogiques ? Pour apporter une réponse à cette question, il aurait fallu susciter un programme d'évaluation coordonné dans les différents sites. Or ce n'est pas le cas : un suivi n'existe actuellement à notre connaissance que dans deux sites ${ }^{10}$, et sans qu'aucune concertation ni verticale, ni horizontale, n'ait été impulsée par le comité de pilotage national de la chaîne.

Le même genre de difficulté se profile avec le programme " Potentialités pédagogiques des réseaux », lancé fin 1989 par la Direction des Lycées et Collèges (D.L.C.), qui a souhaité étendre à cinq nouvelles académies les expérimentations menées dans celle de Bordeaux en matière de câblage d'établissement. Le dispositif de pilotage mis en place à l'échelon national ne prévoit pas d'accompagnement pour le suivi et l'évaluation. Celui-ci est laissé à l'initiative des groupes de pilotage régionaux, qui devront trouver localement les ressources humaines et financières nécessaires en négociant avec des équipes universitaires, ... si elles sont prêtes à s'investir dans ce genre de projet. En tout état de cause, aucune coordination n'est ici encore prévue pour définir les hypothèses de travail sur l'ensemble du programme.

Certes, le caractère mutipartenarial de ces opérations, qui impliquent des acteurs de l'Éducation Nationale, des collectivités territoriales et des acteurs privés, engendre un certain flou quant aux initiatives à prendre. Cette absence de politique concertée de recherche et d'évaluation nous paraît cependant confirmer la difficulté traditionnelle des responsables de l'Éducation Nationale à mettre en place un accompagnement des innovations pédagogiques par des recherches d'une durée suffisante, qui permettraient de tirer des conclusions sérieuses. Les subventions-recherche d'organismes extérieurs (DATAR, Agence du Câble) ne sont le plus souvent accordées que pour le démarrage des opérations, ce qui correspond à la vocation incitatrice de ces instances; mais elles ne sont pas relayées par la suite. 
18 Cette difficulté peut aussi bien sûr être imputée au fonctionnement structurel de la recherche sur le terrain de la F.I., que les futurs I.U.F.M. permettront peut-être de mieux coordonner et de mieux valoriser.

19 En dernière analyse, on peut plus brutalement se demander si la logique actuelle de « marché » qui prévaut dans le paysage audiovisuel français ne tend pas à imposer ses normes dans la sphère publique ${ }^{11}$ : il importerait plus de laisser pénétrer le marché éducatif par les firmes industrielles, que d'engager les recherches nécessaires sur la place des outils dans les stratégies de formation, investigations qui s'inscriraient dans l'histoire déjà longue, faite de quelques succès et de nombreux échecs, de la rencontre des technologies et de l'éducation. Le câblage des établissements serait alors prioritairement l'occasion pour des sociétés spécialisées dans l'ingénierie des réseaux câblés, de mettre au point et de tester des prototypes, et de mobiliser les collectivités territoriales, propriétaires des bâtiments scolaires, pour diffuser l'innovation technique - et cela, avec l'appui des responsables de l'Éducation Nationale ${ }^{12}$.

20 Ainsi, faute d'une volonté politique clairement affirmée de soutenir une recherche accompagnatrice de l'innovation technologique, le secteur de la F.I. n'est pas à l'abri d'une dérive technocratique vers les lois du marché.

21 Qu'en est-il des secteurs qui semblent correspondre à des enjeux plus stratégiques pour les décideurs politiques, et qui bénéficient à ce titre de financements importants ? Quels critères seront mis en avant pour évaluer l'avancée et la réussite des programmes impulsés ? Comment les équipes interdisciplinaires chargées de les mettre en oeuvre font-elles leur place aux approches différentes des chercheurs appelés à collaborer?

22 Apporter des réponses générales à ces questions serait prématuré dans l'état d'avancement des projets, et de toute façon hors de notre portée. Nous nous contenterons d'évoquer les difficultés rencontrées à l'occasion du projet « Outils et méthodes pour la communication avancée ", négocié au départ par sept laboratoires des trois universités lilloises dans le cadre du contrat de plan État-Région, car elles nous paraissent illustrer les conditions faites aux disciplines relevant des sciences sociales.

23 Ce programme, qui s'appuie sur un apport de sciences fondamentales (langages informatiques et représentation des connaissances, résolution de problèmes liés à des scénarios cognitifs) et de sciences appliquées (ingénierie appliquée au domaine de l'éducation et de la santé), affirme clairement sa finalité opérationnelle : la conception d'outils de type hypermédias en vue, dans une seconde phase, de la réalisation d'une plate-forme de communication avancée, comprenant un serveur de connaissances multimédias relié par un réseau de haute performance à des stations de travail spécialisées.

24 Cet objectif correspond bien à la stratégie de la Région, co-décideur et co-financeur avec l'Etat, qui vise à structurer la recherche autour de quelques pôles de développement forts, capables de valoriser une image régionale ${ }^{13}$.

Une année après le lancement effectif de ce programme, les deux laboratoires préoccupés par le maintien d'une dimension anthropologique et critique au sein de celui-ci, s'en trouvent - provisoirement peut-être - écartés. Des raisons liées à des difficultés pratiques et à des problèmes locaux peuvent être invoquées. Mais il faut bien admettre aussi que s'est instauré de fait, dès la négociation initiale, un rapport de force favorable aux gros laboratoires des universités de sciences et techniques et de médecine, habitués à raisonnner en « KF » pour leurs besoins en équipements, et en « masse critique » du point 
de vue du nombre de leurs chercheurs. Cette inégalité structurelle pèse lourd, et même si des recherches fondamentales sont essentielles en amont de la conception des outils, c'est une visée opérationnelle qui prévaut globalement, d'autant que les enjeux symboliques et financiers liés à la réussite du programme sont de taille pour certains des laboratoires engagés (consolidation de leur notoriété nationale et internationale, et donc positionnement favorable pour le projet d'Université Ouverte dans le cadre d'un marché européen des formations).

Une telle coïncidence entre les intérêts des décideurs et ceux des opérateurs de recherche en position dominante, génère des conditions peu propices à une réflexion sur les modalités de cette industrialisation de pointe dans le secteur éducatif : où seront implantées ces stations de travail multimédias conçues pour l'instant en laboratoire? Quelles institutions éducatives se verront dotées de tels systèmes, pour quels besoins, et pour quels types d'usagers ? Comment seront résolus les problèmes à la fois réglementaires et économiques liés à l'usage des réseaux par les différents prestataires de formation ? Et comment sera envisagé le caractère extensif et reproductible d'une telle infrastructure?

Cet ensemble de questions devrait aussi pouvoir trouver des réponses au fur et à mesure de l'avancement du programme et des expérimentations qu'il suscitera en aval. C'est une des conditions pour que le corps social garde la responsabilité publique de ces innovations technologiques, dont on pressent qu'elles vont engager à terme des mutations profondes dans les pratiques et les missions des établissements de F.I., et plus encore probablement des organismes de F.C.

Des négociations sont en cours pour tenter de redonner place à ce type d'interrogations au sein du programme, et déboucheront peut-être sur leur prise en compte lors d'une expérimentation projetée dans le domaine des réseaux câblés appliqués à la formation. Mais rien n'est encore vraiment acquis, malgré le rôle de médiateur de la Région, qui, un peu tardivement, semble intégrer cette dimension de recherche dans ses critères d'évaluation.

\section{Les conditions de la recherche empirique : contraintes liées a l'instabilité des projets.}

\section{Les nouveaux vecteurs comme analyseurs du processus d'industrialisation.}

L'axe de recherche sur l'industrialisation de la formation que nous privilégions ne peut se concevoir sans le substrat empirique que constituent les expérimentations autour de divers vecteurs ou systèmes (réseaux câblés, satellites, réseaux large bande, télématique), qui se caractérisent aujourd'hui par leur intégration de plus en plus poussée. Ceux-ci représentent de véritables analyseurs du processus d'industrialisation. C'est en effet à travers les expériences dont ils sont le support que l'on peut s'attacher à repérer des indices et des tendances d'évolution : ainsi la modification des types de tâches pour les formateurs; la transformation des pratiques des gestionnaires amenés, pour trouver les fonds requis par des équipements coûteux, à rechercher l'appui de nouveaux acteurs, publics ou privés, extérieurs à l'Éducation Nationale ; le cadre imposé aux projets de développement par la logique nouvelle de la contractualisation (projet d'établissement, 
de district, contrat d'agglomération) ; ou encore, la concentration prévisible dans le secteur de la F.C., à cause des investissements lourds qu'entraînent les technologies de pointe.

C'est aussi à travers les expérimentations que l'on peut observer les résistances auxquelles se heurtent ce processus d'industrialisation, du fait des singularités du domaine : ainsi le marché ciblé de la F.C. pose crucialement le problème de la rentabilisation des produits multimédias dont la conception est coûteuse ; en F.I., les contraintes organisationnelles et les normes en vigueur dans les établissements ont toute chance de ralentir la diffusion de scénarios privilégiant l'individualisation et l'autonomisation de la formation ${ }^{14}$.

31 L'identification de ces indices et de ces facteurs de résistance est une opération particulièrement délicate sur le plan méthodologique dans la période actuelle, car les tendances se distinguent encore mal. Il faudrait, pour assurer la validité des indices, pouvoir s'appuyer sur des projets stables, d'une durée suffisante, et dépassant le stade de l'expérimentation si l'on veut étudier les modalités de diffusion des innovations.

Or, ces conditions de la recherche sont difficilement satisfaites aujourd'hui : la mise au point d'une stratégie cohérente est souvent contrariée par des retards dans le démarrage des projets, par des fluctuations dans leur mise en oeuvre, et par des atermoiements au moment où se pose la question de leur extension.

\section{Le démarrage lent des systèmes prototypiques.}

Certains projets intègrent une composante technologique lourde. Nous l'avons vu pour le programme "Outils et méthodes pour la communication avancée »; c'est aussi le cas des projets de câblage d'établissements scolaires. Que leur ingénieries oit confiée à des laboratoires universitaires ou à des sociétés privées, dans les deux cas de figure, les concepteurs sont confrontés à la réalisation de systèmes prototypiques, pour lesquels il n'existe pas, en France ou à l'étranger, de référent transposable tel quel. Il n'est pas étonnant, dans ces conditions, que les délais de mise en service soient souvent beaucoup plus longs que ceux qui sont prévus au départ.

Le calendrier établi pour l'implantation d'un réseau multi-services dans deux lycées de la région retenus dans le programme de la D.L.C. illustre bien cette contrainte : l'élaboration du cahier des charges techniques précisant les caractéristiques attendues, et la réponse en retour de l'opérateur privé, ne devaient pas prendre plus de six mois, ce qui rendait envisageable le démarrage de l'expérimentation au début de l'année scolaire 90-91. De son côté, le groupe de pilotage académique de l'opération avait demandé dès juin à une équipe universitaire interdisciplinaire de présenter pour septembre un protocole de suivi et d'évaluation.

Ce volontarisme paraît bien irréaliste lorsqu'on constate, en ce début d'année 1991, que les travaux n'ont encore commencé dans aucun des deux établissements. Les raisons qui expliquent un tel retard ne sont pas à mettre seulement sur le compte des lenteurs classiques dans tout projet de cette envergure. Il n'est tout simplement pas possible de faire mieux ni plus vite dans cette phase où se négocient des projets qui ont statut de prototypes, avec les nombreux ajustements que cela suppose entre les futurs utilisateurs et l'opérateur chargé de l'implantation technique ${ }^{15}$. De part et d'autre, il faut faire face à une situation dans laquelle les besoins présents et futurs sont encore difficiles à cerner, et 
doivent donc pouvoir être pris en compte au fur et à mesure de leur évolution dans un système souple et modulaire - contrainte qu'il n'est pas facile non plus d'intégrer, du fait que ce système n'est pas implanté dans un bâtiment neuf.

Il ne sera pas davantage possible par la suite, pour l'équipe de recherche, de faire l'économie du temps nécessaire à l'expérimentation elle-même, avant de pouvoir procéder à son évaluation.

\section{Hésitations et incertitudes dans les politiques publiques.}

D'autres projets ne présentant pas les mêmes contraintes techniques, et dont le démarrage n'a pas souffert des mêmes délais, rencontrent dans leur mise en oeuvre des difficultés, du fait d'incertitudes et de fluctuations dans les politiques publiques qui les ont initiés.

Né d'un choix politique fait par le précédent gouvernement, le projet « Pôle national d'enseignement à distance ", confié, fin 1987, aux universités lilloises et valenciennoise, a ainsi vu son financement gelé pendant plus d'un an, par suite de l'arrivée au pouvoir d'une nouvelle équipe qui ne l'a pas immédiatement repris à son compte. De telles hésitations ont eu des retombées inévitables sur le travail des équipes d'enseignants et de chercheurs qui collaboraient à la transformation de deux diplômes de premier cycle universitaire en formation multimédias à distance ${ }^{16}$, sur la base d'un calendrier initial prévu pour une période de quatre ans. Elles ont en particulier contribué à renforcer le défaut de structuration dont a souffert cette opération, qui aurait nécessité la mise à disposition, au moins à temps partiel, d'un responsable de projet, pour aider à concevoir ce nouveau système de formation.

39 Malgré ces aléas, le travail de réalisation et d'évaluation s'est poursuivi, de manière artisanale et à un rythme différent dans les deux universités lilloises, et le stade de l'élaboration des premières maquettes est désormais atteint, au moins dans l'une d'entre elles, pour l'ensemble des modules.

40 Mais de nouveau un blocage se profile au moment où il faut passer à un stade d'industrialisation : les maquettes testées essentiellement jusqu'à présent avec des groupes en face à face, doivent l'être désormais à distance, ce qui suppose de résoudre des problèmes logistiques (équipement en micro-ordinateurs, magnétoscopes, minitels, au domicile des formés ou dans des centres de ressources); la production et la duplication des produits (disquettes, cassettes audio ou vidéo, livrets d'exercices) supposent par ailleurs le recours à des services techniques équipés et performants; enfin, se pose le problème de l'extension du public potentiel de ces DEUG multimédias à distance : ce public excède naturellement les frontières régionales et même nationales, si l'on prend pour référence les actuels usagers du Centre de Télé Enseignement Universitaire (C.T.E.U.).

41 La vocation initiale de ce programme (Pôle national d'enseignement à distance) est donc crucialement à prendre au sérieux à cette étape de son avancement, pour que soient rentabilisés les efforts consentis, qui, ici encore, ont partiellement relevé, du fait des aléas financiers évoqués, de la pratique du bénévolat (cf. note 14).

Or, une perspective claire concernant la formation universitaire à distance en France tarde à se dessiner : bien qu'un rapport ait été commandé par le Ministre de l'Education Nationale sur la création d'une Université Ouverte, et que des conclusions aient été 
remises par son auteur en juin $1990^{17}$, aucune décision n'a encore été prise depuis six mois par les responsables politiques. Cela s'explique bien sûr par le coût financier que représente une telle création ; mais les raisons sont à chercher aussi dans le consensus qu'il faut trouver avec d'autres acteurs impliqués de longue date dans l'enseignement à distance, le CNED et les CTEU.

Toutefois, si les tergiversations et les contradictions ne sont pas résolues à l'échelon national, on voit mal quel sera l'avenir, non pas simplement de ce programme, mais aussi d'autres expérimentations lancées dans d'autres cadres, au sein des universités ou ailleurs, et qui ont besoin de déboucher à terme sur un projet cohérent.

\section{Conclusion} les programmes de certains des laboratoires (d'informatique, de sciences cognitives, d'ingénierie appliquée) impliqués dans les projets, il crée des conditions moins favorables pour les chercheurs soucieux de préserver les exigences critiques de la recherche dans ce secteur-clef, aux avant-postes de la modernisation. La marge de manoeuvre est particulièrement étroite entre la nécessité de coller aux transformations en cours, voire d'y participer aux côtés des décideurs, des professionnels et des praticiens, et la nécessaire prise de distance vis-à-vis du terrain, que suppose la réflexion sur les modalités de l'industrialisation de la communication éducative.

Si cet axe constitue aujourd'hui un domaine d'étude à part entière dans le cadre plus général des recherches sur les industries culturelles au sein des Sciences de la Communication, il lui reste à imposer sa légitimité au sein de ces programmes interdisciplinaires de recherche-développement, comme une composante de leur évaluation. 


\section{BIBLIOGRAPHIE}

Bensaïd, A., (1990), « Nouvelles techniques de communication, complexes multimédias et aménagement des zones fragiles ", Bulletin de l'IDATE, $\mathrm{n}^{\circ}$ 40, pp. 9-24.

Bulletin de l'IDATE, (1986), Éducation, formation et nouveaux médias, $\mathrm{n}^{\circ} 23$.

Bulletin de l'IDATE, (1989), Enseignement et formation. Enjeux et perspectives du développement des médias, $\mathrm{n}^{\circ} 35$.

Chevalier, Y. et Fichez-Vallez, É., (1990), Évaluation de l'expérimentation Éducâble à Grande-Synthe, Document intermédiaire, Académie de Lille, 25 p.

Duhamel, O., (1990), Pour une Université Ouverte, Conclusions à M. Lionel Jospin, Rapport dactylographié, 26 p.

La lettre d'Atena, (1990), Bulletin trimestriel, $\mathrm{n}^{\circ} 4$, Montpellier.

Lam, M., (1987), « DELTA : développement de l'apprentissage en Europe par l'emploi des technologies avancées ", Bulletin de l'IDA TE, nº 26, pp. 48-56.

Lyotard, F., (1979), La condition postmoderne, Paris, Ed. de Minuit.

Moeglin, P., (1990), «L'industrialisation de la formation: dispositifs technologiques et enjeux économiques ", à paraître in Actes du. Colloque CLEMI/INFORCOM 90, Aix-en-Provence, Université de Provence.

Rabaté, F., (1988), "Éducâble ou la télévidéothèque éducative ». Évaluation et stratégie de développement, Montpellier, IDATE.

Ramoff, A., (1990), Introduction des Actes du colloque « Formations multimédias », Paris, La Documentation Française, pp. 9-11.

Schiller, H. I., (1984), Information and the crisis economy, Ablex Publishing Corporation, Norwood, New Jersey.

Singer, D., (1989), « Vers un espace scientifique et technique européen », Bulletin de l'IDATE, n³5, pp. 13-28.

Terroir, P., (1990), Allocution, Actes du colloque « Formations multimédias », Paris, La Documentation Française, pp. 109-110.

\section{NOTES}

1. Ces propos ont été tenus lors du colloque «Formations multimédias : Élémentaire, mon cher Watson! », organisé par la Délégation à la Formation Professionnelle, avec l'appui de la Cité des Sciences et de l'Industrie de la Villette et de Protée, les 25 et 26 janvier 1989.

2. Nous nous référons notamment, pour ce genre d'analyse, aux positions de H.I. Schiller concernant l'économie politique de la communication :

Thus the cultures of the home, the factory, the office, the school and the street, in their utilization of electronics, as if is currently embodied in the new technologies, are, at the same time, adopting commercial modes and networks that integrate and "rationalize " human consciousness, no less than industrial 
production (1984, p.78).

(Ainsi, les cultures du foyer, de l'usine, du bureau, de l'école (souligné par nous) et de la rue, quand elles font place à l'électronique, telle qu'elle est généralement incorporée aux ,nouvelles technologies, en viennent simultanément à adopter les modes et les réseaux commerciaux, facteurs d'intégration et de " rationalisation » de la conscience humaine, au même titre que la production industrielle).

3. L'analyse des conditions de la recherche présentée ici repose largement sur notre expérience dans le domaine concerné. Elle ne prétend donc ni être exhaustive, ni être définitive.

4. Le programme RACE contribue, dans le domaine des télécommunications, à la promotion des communications avancées que DELTA s'efforce d'exploiter pour l'enseignement à distance. COMETT, tout en étant plus un programme de formation que de recherche, s'inscrit cependant dans la lignée des programmes de recherche-développement tels que RACE. Dans son volet D, il prévoit le soutien à des initiatives multilatérales, faisant appel à des systèmes de formation multimédias (cf. pour plus de précisions les articles de M. Lam, 1987, et D. Singer, 1989).

5. Voir la présentation que fait A. Bensaïd (1990) du Centre éducatif intelligent «Ulysse », et de l'appel à projets de février 1990 qui concerne, entre autres, le secteur éducatif.

6. Celui-ci prévoit, dans le cadre de son multipôle technologique régional, le premier d'une série de colloques sur la formation multimédias, en mai prochain.

7. Quelques exemples pour fixer des ordres de grandeur: la phase exploratoire du projet DELTA a entraîné, de 1988 à 1990, une dépense de 40 millions d'Ecus (soit environ 280 millions de Francs) pris en charge à $50 \%$ par la Communauté. L'aide publique se monte à 62 millions de Francs pour le programme multimédias de la D.F.P. Quant au programme régional « Outils et méthodes pour la communication avancée », il s'élève à un montant global de plus de 25 millions de Francs sur 5 ans.

8. Cf. P. Terroir :

(Les formations multimédias) constituent aussi des produits d'exportation que nous devons nous attacher à faire pénétrer sur les marchés de nos partenaires, dans le cadre de la construction européenne notamment (1990, p. 209).

9. Elle est actuellement en service dans 10 sites, et des négociations sont en cours pour une dizaine d'autres.

10. Ces deux sites sont Villeurbanne et Grande-Synthe. Cf. pour Grande-Synthe, le rapport intermédiaire que nous avons rédigé en collaboration avec Y. Chevalier (1990).

11. C'est un argument de ce type que met également en avant $\mathrm{H}$. Dieuzeide, dans l'éditorial de La lettre d'Atena du 4 octobre 1990, lorsqu'il évoque le désengagement des pouvoirs publics vis-à-vis de l'association qu'il préside. Faute de moyens, celle-ci a de plus en plus de mal à remplir les objectifs qu'elle s'était donnés de gestion du programme Olympus, et de recherche sur les nouvelles technologies et la formation à distance, sauf à se reposer sur le bénévolat, voire l'« apostolat » d'un certain nombre de ses membres.

12. On en donnera pour preuve la récente exposition "Ulysse, centre scolaire intelligent ", proposée par l'Académie de Bordeaux et la DATAR, avec le concours de firmes privées (Visicâble, Réseaux câblés d'Aquitaine, Goupil, France-Télécom etc...).

13. L'importance de cette valorisation par des choix prioritaires ciblés transparaît dans les propos du rédacteur d'un document préparatoire à la négociation du contrat de plan :

Il faut que, si l'on pense micro-électronique, hyperfréquences, ou parasitologie, on pense immédiatement Nord-Pas-de-Calais.

14. Ces propositions font l'objet de développements dans notre dossier d'habilitation à diriger des recherches (Université de Lille 3, 1991). P. Moeglin (1990) analyse de son côté d'autres facteurs constituant autant de résistances fortes à l'extension des modèles industriels, tels que la pratique du bénévolat, répandue aussi bien en F.I. qu'en F.C. 
15. La société opératrice Région-Câble, restée seule en lice pour le marché, a été amenée, dans un court laps de temps, à se structurer pour répondre à ces besoins nouveaux en services professionnels émergeant du secteur éducatif, mais aussi hospitalier et industriel. Elle l'a fait en créant un bureau d'études ensemblier "Interact», chargé de la conception et de la mise en oeuvre de " produits-services ", façonnés sur mesure, selon les besoins des futurs utilisateurs.

16. Les deux diplômes concernés sont le DEUG Langues Étrangères Appliquées à l'Université des Lettres et Sciences Humaines de Lille III, et le DEUG Sciences et Structures de la Matière, à l'Université des Sciences et Techniques de Lille I.

17. Il s'agit du rapport de O. Duhamel, « Pour une Université Ouverte ».

\section{RÉSUMÉS}

Dans la phase présente d'industrialisation des services éducatifs, les appels d'offre de décideurs/ financeurs nationaux et européens se multiplient, créant ainsi d'apparentes conditions favorables pour le développement de la recherche dans ce secteur. Toutefois, on peut craindre que l'exigence de performativité requise des équipes prenant en charge ces programmes ne favorise surtout la mise au point de dispositifs de travail ou d'outils, au détriment de recherches visant à anticiper et à évaluer les usages éducatifs de ces systèmes et produits. Les modalités de l'industrialisation du secteur éducatif risquent ainsi d'échapper à une réflexion critique, d'autant plus que les pratiques de recherche à finalité non opérationnelle se trouvent contrariées à la fois par les conditions même de l'émergence et de la diffusion de l'innovation technique, et par l'instabilité des politiques publiques. Cette situation ne doit cependant pas faire renoncer au maintien d'une visée critique, ouvrant la voie d'un contrôle du corps social.

In the present period of industrialized educative services the calls from executive and financial circles on a national and European scale are multiplied, thus creating apparently favorable conditions for the development of research in that field. However, one can fear less the ability of performance required from these research teams might be chiefly favorable to the elaboration of patterns or systems of working, to the prejudice of a research which would aim at anticipating or estimating the way these patterns and systems are used for education. There is a risk that the processus of industrialization in the educational field might escape a critical reflection especially as the practice of non-operationnal research would be thwarted both by the appearance and circulation of new technicalities and by unsteady public policies. In spite of that situation we mustn't give up a critical view, opening up the way to a control by ail citizens.

\section{INDEX}

Mots-clés : politique de la recherche, Nouvelles Technologies Éducatives (NTE), multimédia en éducation secteur éducatif, industrialisation

Keywords : politics of research, New Educational Technologies (NET), multimedia in education, educational sector, industrialization 
AUTEUR

ÉLISABETH FICHEZ-VALLEZ

Élisabeth Fichez-Vallez, Université Charles de Gaulle-Lille III. 